\title{
Pediatric heart transplantation: long-term outcomes
}

\author{
Anne I. Dipchand ${ }^{1}$ - Jessica A. Laks ${ }^{1}$ \\ Received: 17 October 2018 / Revised: 15 March 2019 / Accepted: 19 March 2019/Published online: 29 May 2019 \\ (C) Indian Association of Cardiovascular-Thoracic Surgeons 2019
}

\begin{abstract}
Pediatric heart transplant has become the standard of care for end-stage heart disease in children throughout the world. The number of transplants has grown dramatically since the first transplant was performed, and over the last two decades, outcomes have consistently improved with progression in knowledge enhancing the clinical course and outcomes of these patients. Shortterm outcomes in the most recent era have been excellent resulting in a renewed focus towards medium- and long-term outcomes. This article will review the most up-to-date literature on overall heart transplantation outcomes and specific long-term outcomes including rejection, cardiac allograft vasculopathy, graft failure, infection, renal dysfunction, malignancy, and the need for retransplantation. The article also explores the post-transplantation outcomes of special populations, including Fontan patients, ABO-incompatible recipients, sensitized recipients, extracorporeal membrane oxygenation, and ventricular assist devices. The article concludes with a look at transition from pediatric to adult care and medication adherence, which are becoming major issues related to long-term outcomes as post-transplant survival increases.
\end{abstract}

Keywords Pediatric · Transplant · Outcomes

\section{Introduction}

Pediatric heart transplant has become the standard of care for end-stage heart disease in children throughout the world. The number of transplants has grown dramatically since the first transplant was performed in 1967 with the annual number of transplants reported to the International Society of Heart and Lung Transplantation Registry increasing from 414 reported in 2000 to 684 reported in 2015, with almost 14,000 transplants in children reported in total. [1] Over the last two decades, outcomes have consistently improved with progression in knowledge enhancing the clinical course and outcomes of these patients. Short-term outcomes in the most recent era have been excellent resulting in a renewed focus towards medium- and long-term outcomes. Rejection, cardiac allograft vasculopathy (CAV), graft failure, and infection continue to be the major causes of death within the first 5 years post-transplant. [2] As well, renal dysfunction, malignancy, and the need for re-transplantation remain as significant issues that require

Anne I. Dipchand

anne.dipchand@sickkids.ca

1 Cardiology and Transplant, Institution: Hospital for Sick Children, 555 University Avenue, Toronto, Ontario M5G 1X8, Canada close follow-up. This article will review the most up-to-date literature on overall heart transplantation outcomes, as well as take a closer look at specific long-term outcomes, and explore the post-transplantation outcomes of special populations, including Fontan patients, ABO-incompatible recipients, sensitized recipients, extracorporeal membrane oxygenation (ECMO), and ventricular assist devices (VAD). The article will conclude with a look at transition from pediatric to adult care and medication adherence, which are becoming major issues related to long-term outcomes as post-transplant survival increases.

\section{Data sources/registries and collaborations}

Despite pediatric heart transplant becoming the standard of care for end-stage heart disease in children, there still remains a small number of patients that follow this trajectory. Therefore, it is a challenge to acquire the information needed to develop best practices and to study the effects of new diagnostic or therapeutic approaches on outcomes.

There are, however, two main sources of multicenter data for children following listing for heart transplantation and after transplantation, the registry of the International Society of Heart and Lung Transplantation (ISHLT) and the Pediatric Heart Transplant Society (PHTS). As well, there are many 
single-center reports and smaller collaborations. The ISHLT registry is an international registry; in the USA, all data from the United Network of Organ Sharing (UNOS) is shared with the database, as is all European data from Eurotransplant; there are several other smaller collectives and otherwise multiple single centers submitting data. With almost 14,000 transplants in children reported in total, it is felt to represent three quarters of the worldwide transplant activity (https://www. ishlt.org/registries/ttx-registry). The PHTS, on the other hand, is a voluntary, research-based, and event-driven multicenter registry that was established in 1993 in order to capture data relative to outcomes, and currently includes 8681 patients listed for transplantation of which 6524 have undergone transplantation between January 1, 1993 and May 1, 2018 from 55 participating centers in North America, the United Kingdom, and Brazil (https://www.uab.edu/medicine/phts/).

\section{Overview of pediatric heart transplant}

\section{Indications and contraindications}

Primary diagnoses and indications for pediatric heart transplantation have evolved over time. Congenital heart disease has remained the most common underlying diagnosis in infants younger than 1 year of age; however, the proportion of infant recipients with cardiomyopathy has almost doubled in the most recent era. [2] Cardiomyopathy remains the most common indication for transplant in older children. Primary diagnoses and indications also show geographic variation with congenital heart disease and re-transplantation being more common in North America compared with Europe and the rest of the world.

The evaluation for candidacy for heart transplantation includes careful work-up for potential contraindications; however, for the most part, these contraindications are not absolute and the decision regarding transplant eligibility should be made on the individual basis. Potential contraindications include severe or irreversible pulmonary hypertension; obesity; malignancy; diabetes with end-organ damage; renal dysfunction; peripheral vascular disease; active infection; severe pulmonary or liver disease; chromosomal, neurologic, or syndromic abnormalities; active tobacco and substance abuse; and psychosocial issues. [3]

\section{Surgical technique}

Coordinating a pediatric heart transplant is complex and unlike other solid organs. The cardiac graft is extremely susceptible to ischemia, especially when the ischemic time exceeds 4 to 6 hours. Standard operative technique includes both the biatrial and bicaval approaches. [4] The bicaval approach aims to preserve the donor right atrium resulting in several advantages that impact 30-day and long-term survival including preservation of right atrial function, reducing incidence of late atrial dilation, reducing tricuspid regurgitation, and reducing the number of atrial arrhythmias. [5] It has therefore become the surgical technique used in the majority of pediatric heart transplants. [4]

\section{Overall post-transplant outcomes}

The ISHLT registry's most recent analysis shows that the longest survival is for those patients $<1$ year of age at transplantation with a median survival of 22.3 years, followed by 18.4 years for those ages 1 to 5 years, 14.4 years for those 6 to 10 years, and 13.1 years for adolescents (Fig. 1). With the greatest risk of death being in the first-year post transplant, those who survived the first year had a median survival of $>$ 15 years in all age groups. [1] Over time, there has continuously been an era effect, with patients transplanted in more recent eras having better survival compared to earlier eras (Fig. 2); however, the magnitude of this effect has been decreasing and is not evident for all age groups. Males and females had similar survival, and there was no difference in survival based on donor/ recipient gender match or mismatch. [1]

With the demand for organs greatly exceeding the supply, it is necessary to consider 'marginal' donors including those who are older and larger. Historically, concern was raised regarding weight mismatch and its effect on mortality, rightventricular failure, left-ventricular failure, and hypertension. However, more recent literature has shown that increasing donor-recipient weight mismatch in pediatric heart transplantation does not adversely affect outcomes and in fact may be beneficial. A study out of Great Ormond Street looked at 161 patients undergoing heart transplantation between 2000 and 2008 and divided them into three groups based on their donorrecipient weight ratio. [6] Donor-recipient body weight ratio was analyzed with respect to intubation time, time in intensive care unit, development of right-ventricular failure, mediumterm survival, and freedom from medium-term hypertension. On linear regression, mean intubation time and mean time in the intensive care unit were not related to donor-recipient weight ratio. Nearly $19 \%$ of patients in the lowest donorrecipient weight ratio group developed right-ventricular failure compared to $24.5 \%$ in the middle group and $28.8 \%$ in the upper group of weight mismatch $(p=0.48)$. On survival analysis, mortality was highest among those with the lowest donor-recipient weight ratio $(p=0.04)$; however, there was no difference in midterm survival or condition of survival to discharge $(p=0.14)$. There was also no association between weight ratio and freedom from medium-term hypertension $(p=0.39)$. There was no association between the extreme mismatch group of $3 \times$ or greater and either midterm mortality $(p=0.76)$ or freedom from hypertension $(p=0.62)$, but this 
Fig. 1 Kaplan-Meier survival curve out to 25 years after pediatric heart transplantation stratified by age at the time of transplantation (used with permission from the Registry of the International Society of Heart and Lung Transplantation. J Heart Lung Transplant 2017;36:103779)

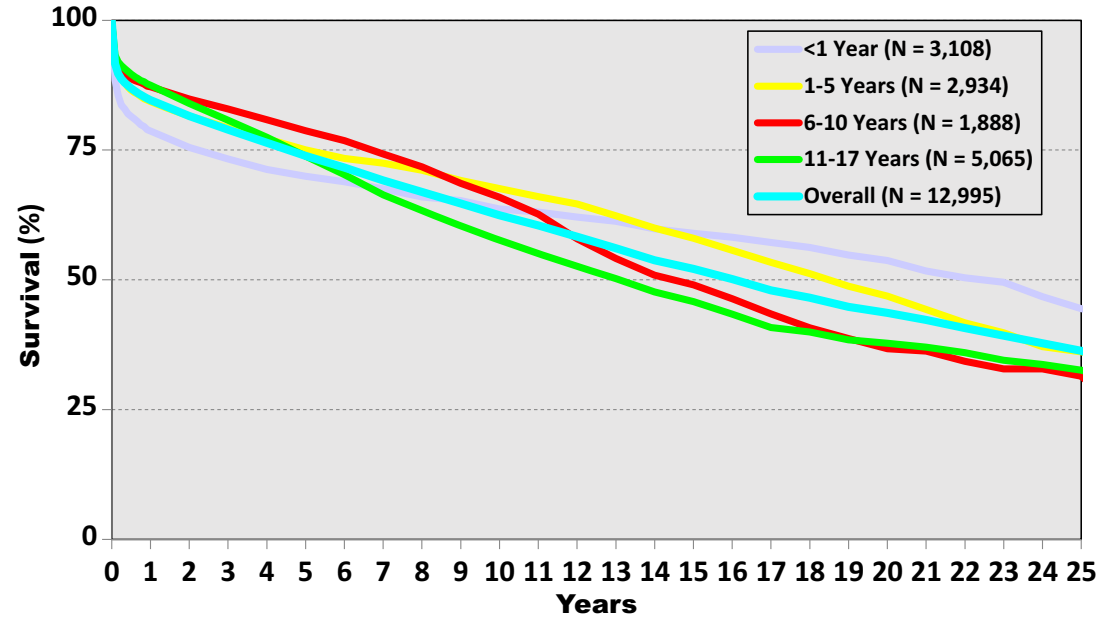

was associated with the need for post-operative ECMO, support $(p<0.01)$. [6]

Diagnosis before transplantation also affects survival with patients with congenital heart disease having significantly lower intermediate-term survival after transplant when compared to patients with dilated cardiomyopathy. Although historically, single ventricle patients with prior staged surgical palliation fared the worst, recent data actually shows a significant improvement in post-heart transplant survival in Fontan patients. [7] Myocarditis is associated with a worse post-transplant outcome with a survival of $83 \%$ at 1 year and $65 \%$ at 3 years post-transplantation. [8] Factors that have been shown to affect 1-year mortality include ECMO, being on a ventilator or dialysis at the time of transplant, other markers of renal insufficiency (creatinine and glomerular filtration rate), recipient body mass index, recipient total bilirubin, ischemic time, and transplant center volume. [1, 2] Graft failure, rejection, infection, and CAV continue to be the major causes of death within the first 5 years post-transplant (Fig. 3).

\section{Rejection}

Acute rejection remains a significant cause of morbidity and mortality after transplantation. [9] Multiple analyses continue to demonstrate a relationship between early rejection, cumulative episodes of rejection, and late rejection as risk factors for poor outcome, including death, re-transplant, and CAV. [10] The risk of rejection remains the highest in the first-year post-transplant; however, recent data from the PHTS shows that the incidence of rejection declined from 1993 to 2005 from 60 to $40 \%(p<0.001)$, as did the number of rejections per patient. [11] ISHLT also showed similar findings in the percentage of patients treated for rejection between hospital discharge and 1-year post-transplant from 2004 to 2015 across all age groups and genders (Fig. 4). There are few known identifiable risk factors for rejection other than sensitization; however, induction therapy has not been found to decrease the likelihood of treated rejection (Fig. 5).
Fig. 2 Kaplan-Meier survival curve out to 25 years after pediatric heart transplantation stratified by era (used with permission from the Registry of the International Society of Heart and Lung Transplantation. J Heart Lung Transplant 2017;36:103779)

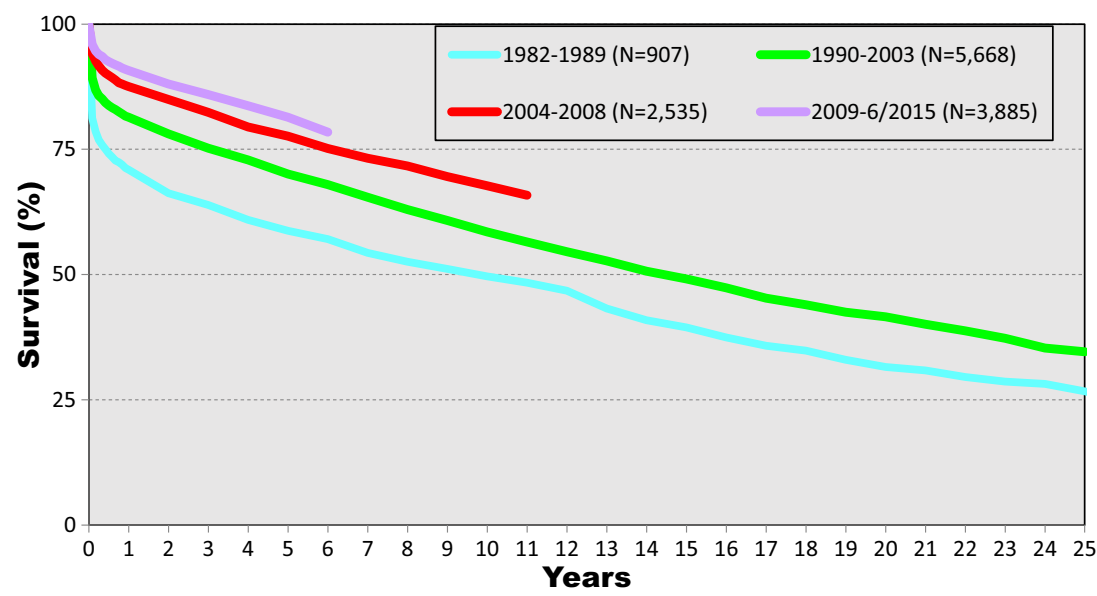


Fig. 3 Relative incidence of the leading causes of death for the most recent era January 2004 to June 2016 following pediatric heart transplant (used with permission from the Registry of the International Society of Heart and Lung Transplantation. J Heart Lung Transplant 2017;36:103779)

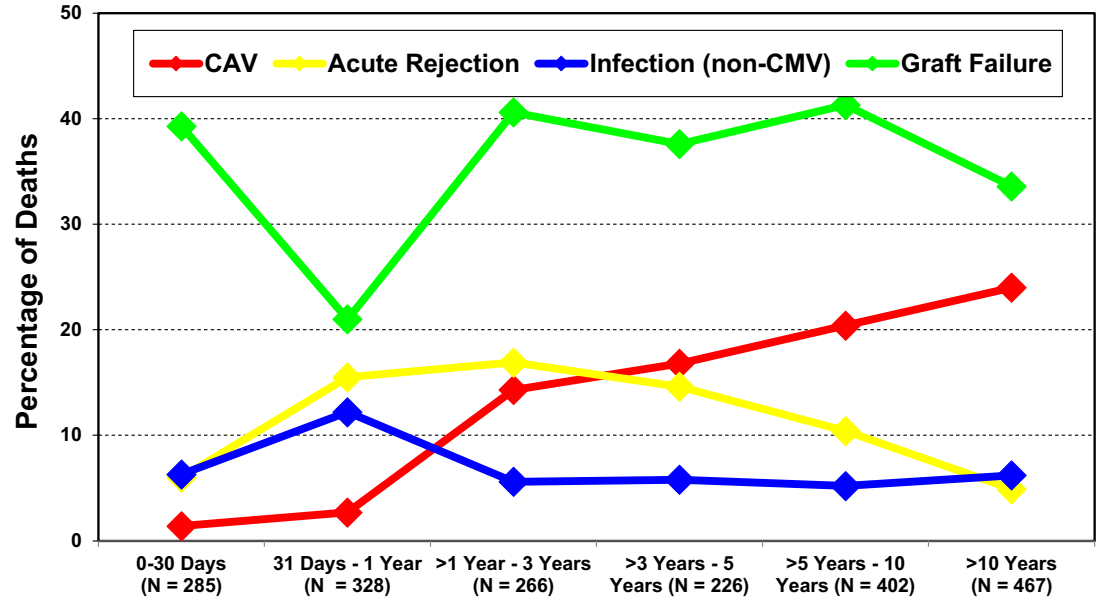

\section{Late rejection}

Similar to early rejection, late rejection or rejection beyond 1 year post-transplant has also decreased in the current era with a PHTS analysis demonstrating an overall decrease in both first and recurrent late rejection. [10] Late rejection was also found to be significantly associated with early rejection $(p<0.001)$ across all eras and recipients with late rejection after early rejection had significantly increased risk of developing moderate to severe CAV $(p<0.001)$. Additional independent risk factors for late rejection included earlier era, nonwhite race, older recipient age, early rejection with hemodynamic compromise, recipient without congenital heart disease, and male donor.

\section{Surveillance}

Endomyocardial biopsy (EMB) remains the mainstay for the diagnosis of rejection. However, there remains a wide variation in the utilization of surveillance EMB among pediatric heart transplant centers. [12] Fewer EMB are performed in infants compared to older patients; however, there has been a shift towards less reliance on EMB in the current era for adolescent patients. As well, when compared to centers within the USA, international centers perform significantly fewer surveillance EMB in adolescents. The etiology of the variation in the use of surveillance EMB remains unclear; however, differences in clinical exposure and mentorship during training as well as programs developing as an extension of more invasive adult programs remain as viable rationalizations. This points to the need for further research to determine the optimal frequency of invasive monitoring without compromising outcomes.

A decline in the incidence of rejection in the current era [11] has led to many questioning the continued utility of routine invasive testing. Many efforts are underway to identify and validate non-invasive tests including echocardiography, intramyocardial electrography, and profiling of gene expression; however, to date, no non-invasive test has been developed to consistently and accurately diagnose and/or predict rejection. [2]

\section{Treatment}

Treatment for acute rejection is dependent on multiple factors including the type, grade, time post-transplant, clinical and
Fig. 4 Kaplan-Meier survival in pediatric heart transplant recipients based on the presence of rejection within the first year after transplantation (conditional on survival to 1 year) (used with permission from the Registry of the International Society of Heart and Lung Transplantation. J Heart Lung Transplant 2017;36:103779)

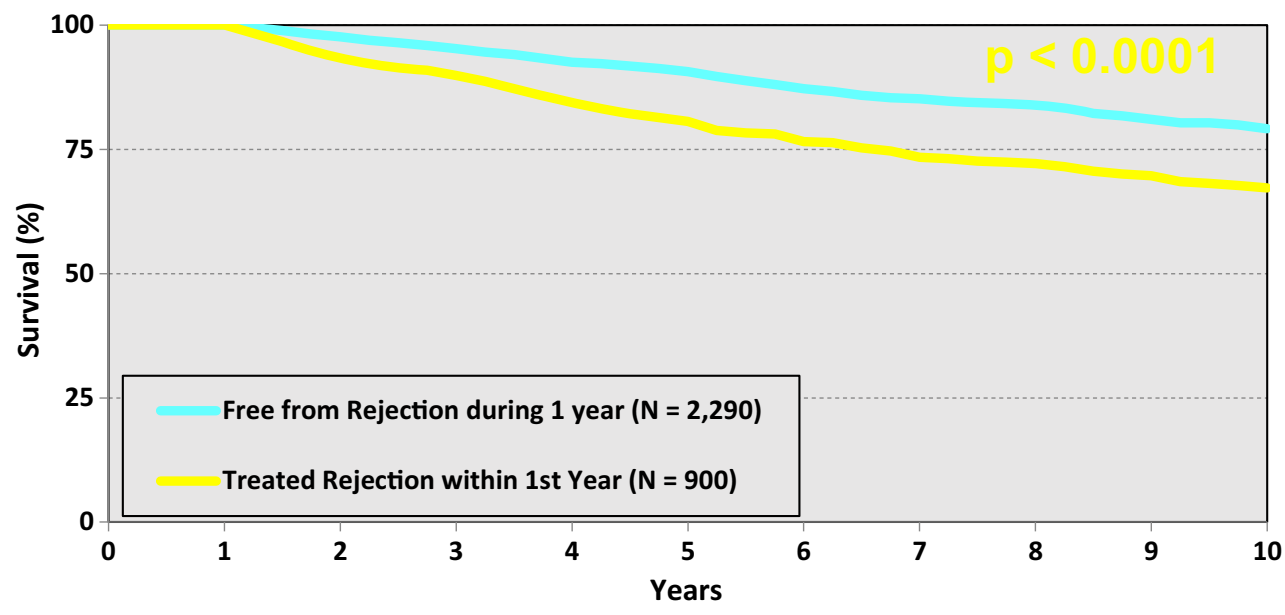


Fig. 5 Percentage experiencing treated rejection between discharge and 1-year follow-up by induction in pediatric heart transplant recipients (used with permission from the Registry of the International Society of Heart and Lung Transplantation. J Heart Lung Transplant 2017;36:103779)

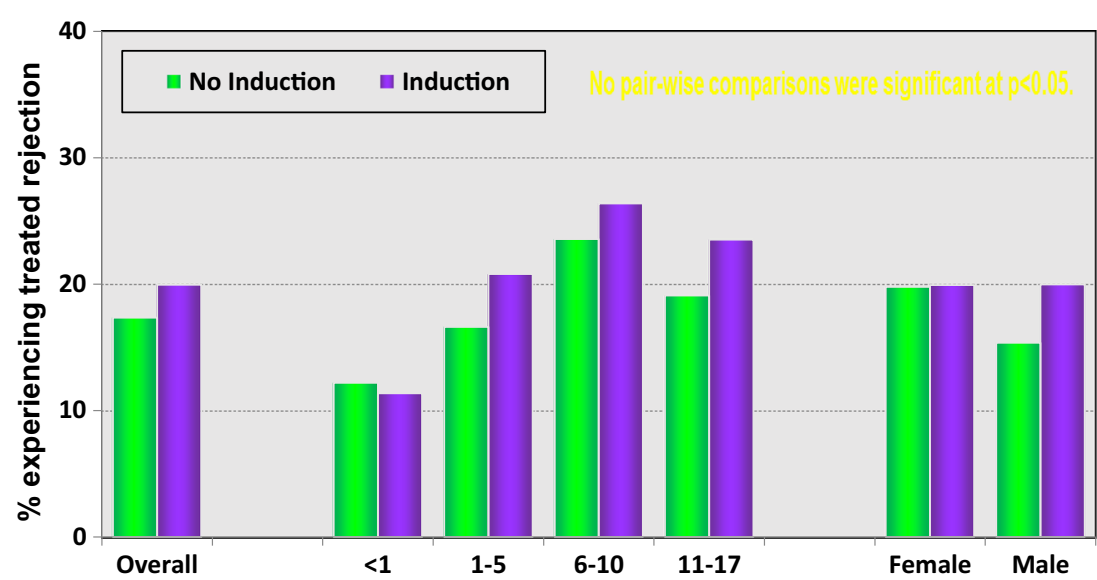

hemodynamic effect, maintenance immunosuppression, comorbidities, and potential co-factors. [2] Mild rejection (ISHLT Grade $1 \mathrm{R}$ ) does not generally require specific intervention, and moderate rejection (ISHLT Grade 2 R) usually requires some degree of increased immunosuppression, including an oral or intravenous corticosteroid and increase in maintenance immunosuppression.

\section{Cardiac allograft vasculopathy}

Cardiac allograft vasculopathy (CAV) is one of the leading causes of morbidity and mortality in heart transplantation and is the leading cause of death beyond 3 years posttransplantation (Fig. 3). [2, 9] A 2013 study estimated the incidence of CAV to be $13 \%$ at 5 years, $25 \%$ at 10 years, and $54 \%$ at 15 years after transplant. [13] Factors significantly associated with shorter CAV-free survival in the study were older recipient age, donor age $>18$ years, re-transplantation, recipient black race, and donor cigarette use. In the ISHLT's analysis, factors associated with the development of CAV included earlier transplant era, no induction therapy, older age at transplant, and rejection in the first year post-transplant. [1] A PHTS report analyzing data from 751 children who had coronary angiograms after heart transplant demonstrated incidence of angiographic coronary abnormalities at 1,3 , and 5 years of $2 \%, 9 \%$, and $17 \%$, respectively. [14] Older recipient age, older donor age, and two or more episodes of rejection in the first year were found as risk factors for CAV.

Graft survival after the diagnosis of CAV has remained low and differed by age groups with infants having the poorest graft survival after the diagnosis (median of about 2 years). [1] Pahl et al. found that overall freedom from graft loss caused by primary CAV was $99 \%, 96 \%$, and $91 \%$ at 1,5 , and 9 years, respectively, after transplantation; however, $24 \%$ of patients with any CAV died within 2 years, and $50 \%$ of patients with moderate-to-severe CAV died within 2 years. [14] Kobayashi et al. showed an estimated graft survival after
CAV development of $42 \%$ at 5 years, $24 \%$ at 10 years, and $16 \%$ at 15 years with an initial drop off at time 0 , indicating that approximately $23.5 \%$ of patients had CAV diagnosed and graft failure simultaneously. [13]

Prevention of CAV is hypothetically a means to improve long-term graft and patient survival. Transplant recipients with known risk factors for CAV should be closely monitored and receive early intervention to reduce CAV risk. Statins have been shown to play a beneficial role in slowing the development of CAV with adult studies showing a benefit on the incidence of acute rejection with hemodynamic compromise, improved 1-year survival, and reduced development of CAV. [2] A 2002 study looking at the efficacy and safety of atorvastatin after pediatric heart transplantation found the use of atorvastatin to be effective in lowering cholesterol, triglyceride, and LDL without altering HDL levels. Complications were rare and included rhabdomyolysis in $5 \%$ of cases. [15] Given the high prevalence of lipid abnormalities in this population, the authors felt that prophylactic use of statins was indicated early after heart transplantation; however, specific evaluation into the role of statins against $\mathrm{CAV}$ was not done in this study. A 2005 study reviewing the use of pravastatin therapy in patients after pediatric heart transplantation found that pravastatin therapy was associated with a statistically significant reduction of CAV ( $p=0.03)$. [16] In the PHTS analysis, however, statin therapy did not confer a survival benefit and was not associated with delayed onset of CAV. [17] In fact, patients receiving a statin had a significantly higher incidence of rejection after 1 year of follow-up $(p=0.0008)$, a significantly increased risk for rejection requiring treatment $>1$ year post-transplant if they had experienced earlier rejection $(p=$ 0.0046), and a trend towards an increased risk of late rejection in those without early rejection $(p=0.0596)$. However, despite the scarcity of pediatric data, statin use continues to be incorporated into the majority of maintenance protocols for pediatric heart transplant recipients.

Since the advent of mTOR inhibitors, such as sirolimus and everolimus, there have been multiple studies looking into their 
ability to treat or prevent $\mathrm{CAV}$ in adult heart transplant recipients with mixed results. Two large randomized controlled trials found that both everolimus and sirolimus, respectively, were superior to azathioprine in preventing $\mathrm{CAV}$ and demonstrating significantly less medial and intimal thickening. [18, 19] However, a recent study of 111 adult heart transplant patients with established CAV who were previously managed with calcineurin-inhibitor and either MMF or azathioprine and were randomized to receive everolimus as a third agent or continue their current two-drug protocol showed no difference in intimal thickness at 1-year follow-up. [20] The side effect profile of mTOR inhibitors is significant and includes impaired wound healing, pulmonary fibrosis, synergistic renal toxicity, hyperlipidemia, painful mucosal ulcers, and increased risk of bacterial and fungal infections among others. Given this and the mixed results of the effect of mTOR inhibitors on prevention of CAV as well as the limited data in pediatrics, the potential routine use of these medications in children requires further study. [21]

Intervention for $\mathrm{CAV}$ is challenging and there is limited experience reported in pediatrics. A study using PHTS data looked at the associations between percutaneous revascularization procedures (PRPs) and outcomes after heart transplant in pediatric patients who received a first heart transplant between 1993 and 2009. [22] Revascularization procedures were done in less than $1 \%$ of patients at a mean time of $5.7 \pm 3.2$ years post-transplant. Vessels involved were left anterior descending artery in $41 \%$ of cases, right coronary artery in $25 \%$, circumflex artery in $18 \%$, and other coronary branches in $16 \%$. PRPs consisted of stent implantations in $75 \%$ of patients and balloon angioplasties in $25 \%$ of patients. Overall procedural success rate was $73 \%$. Freedom from graft loss after PRPs was $89 \%$, $75 \%$, and $61 \%$ at 1,3 , and 12 months. Patients with transplants from donors $>30$ years old were found to have less freedom from the need for a PRP than patients with transplants from younger donors $(p<0.0001)$. The significant graft loss reported within 1 year post-procedure reaffirms that ultimately, patients with moderate-to-severe $\mathrm{CAV}$ and evidence of graft dysfunction should be considered for re-transplantation.

\section{Renal dysfunction}

Late renal dysfunction after heart transplantation in children continues to be a potential risk with a small proportion of children progressing on to dialysis and/or renal transplant (Fig. 6). The likelihood of severe renal dysfunction, dialysis, or renal transplant increased over time after transplant. [1] Freedom from severe renal dysfunction was lowest in patients 6 to 10 years old and adolescents and highest in infants. Freedom from renal replacement therapy was also associated with age at time of transplant with older patients having an increased need for renal replacement therapy. Data from PHTS showed freedom from late renal dysfunction of $71 \%$ and $57 \%$ at 5 and 10 years, respectively. [23] Risk factors for late renal dysfunction from the PHTS study included earlier era of heart transplantation, black race, rejection with hemodynamic compromise in the first year after heart transplantation, and lowest quartile eGFR at 1 year post-heart transplantation. Interestingly, renal function at the time of heart transplantation was not associated with onset of late renal dysfunction.

\section{Infections}

Infections remain a significant challenge and major cause of morbidity and mortality across the entire post-transplant period. Given the source of the majority of the published English literature, the infections discussed herein are reflective of the North American and European experience. In pediatric heart transplant recipients, infections account for $14 \%$ of deaths in the first year after transplant and remain among the top 5 causes of death in subsequent years. [1] This number has
Fig. 6 Freedom from severe renal dysfunction by age group in pediatric heart transplant recipients (used with permission from the Registry of the International Society of Heart and Lung Transplantation. J Heart Lung Transplant 2017;36:103779)

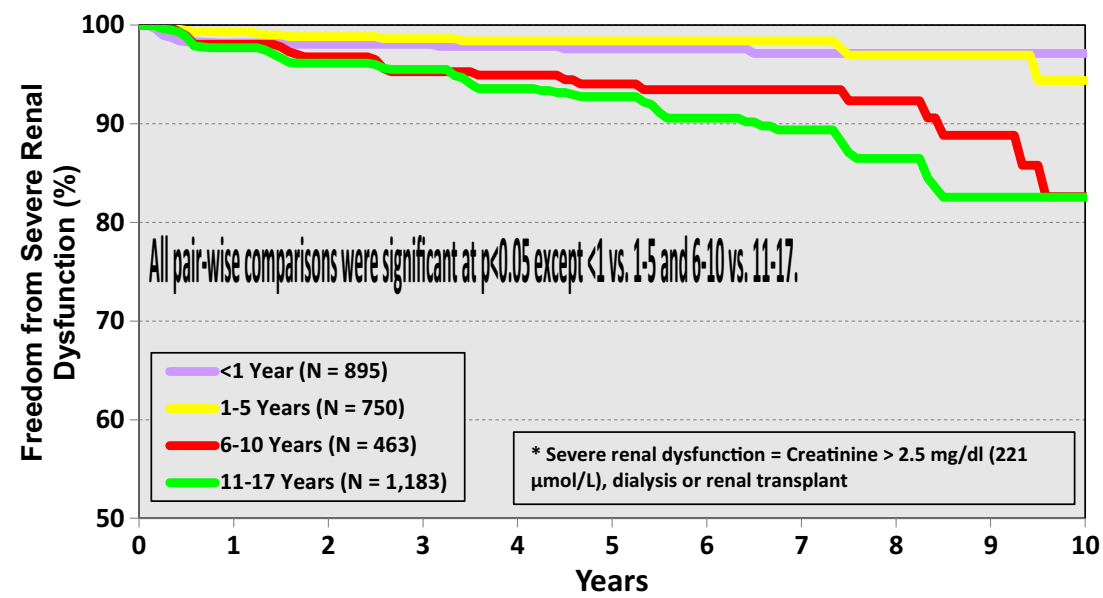


increased from ISHLT's 2015 report, where infections accounted for only $9.7 \%$ of deaths in the first year after transplant. [24] Bacteria are the most common etiology of these infections, affecting up to half of all pediatric heart transplant recipients. PHTS data showed that the risk of bacterial infection was highest in the first month post-transplant and the bloodstream was the most common site. [25] In the early post-transplant period of $<30$ days post-transplant, coagulase-negative staphylococci were the most common pathogens followed by Enterobacter sp. and Pseudomonas sp. In the late post-transplant period, however, communityacquired pathogens such as Streptococcus pneumonia and Haemophilus influenzae were more commonly identified. The same PHTS data identified risk factors associated with the acquisition of bacterial infection including younger age $(p<0.0001)$ and ventilator $(p<0.0001)$ or $\operatorname{ECMO}(p=0.03)$ use at time of transplant. Mortality post-bacterial infection was as high as $34 \%$ with previous cardiac surgery $(p<0.001)$ and multiple sites of infection $(p=0.004)$ as independent predictors of death. [25] Though there is a paucity of published data, there is a wealth of clinical experience with region-specific infectious considerations in South America, India, and Australasia that is beyond the scope of this chapter.

In the past, cytomegalovirus (CMV) infection had been implicated in a number of post-transplant complications including increased risk of developing CAV and death. However, this was not demonstrated in a PHTS analysis to determine the impact of recipient CMV status and CMV mismatching on post-transplant outcomes and the influence of prophylaxis. [26] Freedom from clinical CMV infection was $91 \%$ at 5 years posttransplant with an increased risk in the setting of CMV mismatch. Pre-transplant CMV serology was not associated with mortality $(p=0.40)$ or risk of developing CAV $(p=0.10)$, nor was the use of CMV prophylaxis. There was also no significant association between CMV prophylaxis and the development of clinical CMV infection. Despite this, CMV infection does remain one of the most common complications affecting solid organ transplant recipients including heart transplants. Therefore, international consensus guidelines have recently been updated to direct CMV management including prevention, treatment, diagnostics, immunology, drug resistance, and even pediatric-specific issues. [27]

Pneumocystis jiroveci (PJP) is another transplantrelated opportunistic infection for which widespread prophylaxis is common. A retrospective cohort study using data from PHTS found $1 \%$ of pediatric heart transplant recipients in the database with PJP. [28] The majority of PJP occurred 2 months to 2 years posttransplant, and patients with PJP had significantly decreased mortality compared with other fungal infections. Percent freedom from PJP for pediatric heart transplant recipients was $98.8 \%$ at 10 years, and PJP was the cause of death in $0.05 \%$ of the entire patient population. An updated international consensus guideline on PJP prophylaxis is currently being developed.

\section{Malignancy}

The majority of malignancies occurring in pediatric heart transplant recipients in the ISHLT registry are lymphomas with skin cancers being extremely rare. Freedom from malignancy was $98 \%$ at 1 year post-transplant and $89 \%$ at 10 years posttransplant and was similar across all age groups (Fig. 7). There was no association with type of calcineurin inhibitor or use of induction therapy for freedom from lymphoma (Fig. 8). [1]

Of the lymphomas, post-transplant lymphoproliferative disorder (PTLD) remains the major barrier to long-term survival post-heart transplantation in pediatrics. Early PHTS data reported $5 \%$ of pediatric heart transplant recipients developing PTLD with a probability of survival of $75 \%$ at 1 year, $68 \%$ at 3 years, and $67 \%$ at 5 years after diagnosis. [29] More recent PHTS data looking at the incidence and hazard for PTLD demonstrated similar findings to the ISHLT data with an overall freedom from PTLD of $98.5 \%$ at 1 year, $94 \%$ at 5 years, and $90 \%$ at 10 years. [30] Of the malignancy events reported in this analysis, 97\% were classified as PTLD. Children between the ages of 1 and 9 years and positive donor EBV status in a seronegative recipient were strong risk factors for PTLD. Almost $25 \%$ of EBV seronegative recipients of EBV positive donors at ages 4 to 7 at transplantation developed some form of PTLD. However, when PTLD as a function of age at time of transplantation was stratified by year of transplant, there was a reduction in risk of PTLD with each succeeding era in EBV seronegative recipients of EBV-positive donors, demonstrating an overall decrease in PTLD risk in the most recent transplant era. That being said, it remains a challenge to diagnose and treat with high mortality and relapse rates; thus, international practice guidelines are in the process of being developed and expected in 2019.

\section{Re-transplantation}

Re-transplantation makes up a very small proportion of heart transplants in children annually with the percentage of retransplants remaining relatively stable over time, comprising $5 \%$ of all pediatric heart transplants in 2015 (Fig. 9). Most retransplants occurred at $>60$ months from the primary transplant and occurred among patients $>5$ years old (Fig. 10). [1]

In 2006, PHTS published the first registry-based study examining the incidence and outcomes of pediatric re-transplantation. [31] The most common indication for a second transplant was CAV followed by rejection-related graft failure, 
Fig. 7 Freedom from malignancy by type of malignancy in pediatric heart transplant recipients (used with permission from the Registry of the International Society of Heart and Lung Transplantation. J Heart Lung Transplant 2017;36:1037-79)

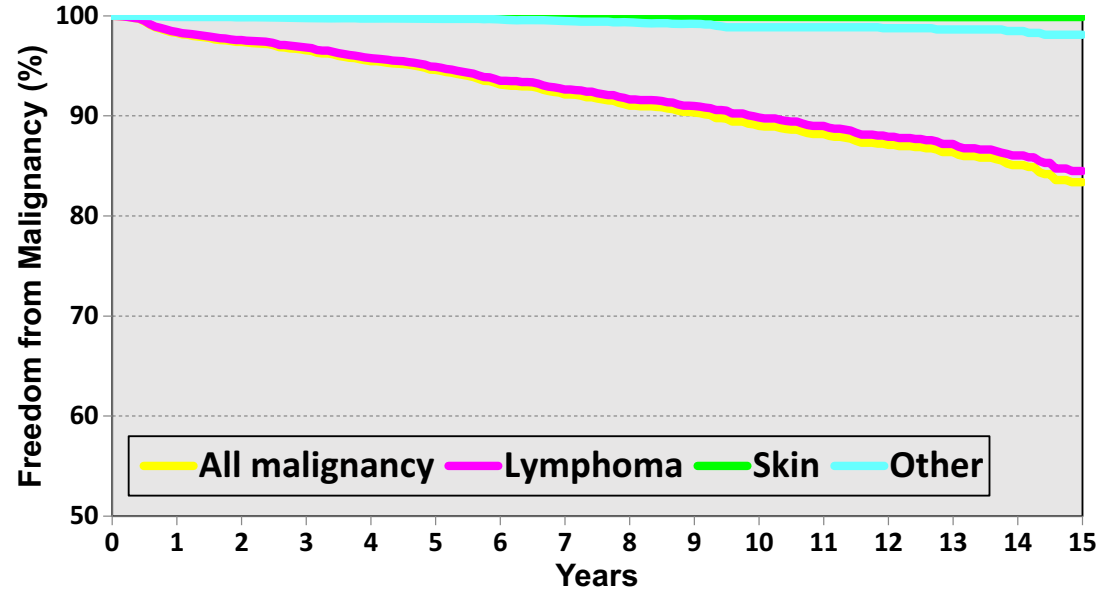

early graft failure, and other/unknown causes. The need for ventilator support, African American ethnicity, and an elevated creatinine at time of transplantation were found to be risk factors for re-transplantation. Patient survival was worse to that after primary transplantation with $80 \%$ at 1 year, $69 \%$ at 3 years, and $60 \%$ at 5 years post re-transplantation $(p=0.04)$. Outcomes were influenced by indication for re-transplantation, with those undergoing re-transplantation for CAV showing the best survival probability and those re-transplanted for early graft failure fairing quite poorly with significant early mortality. [31]

A more recent detailed analysis from the ISHLT registry described outcomes after re-transplantation compared with primary transplantation, including identifying risk factors leading to re-transplantation and transplant-related morbidity and mortality. [32] Similarly to the PHTS data, the most common indication for re-transplantation was CAV (58.5\%) with these patients having the highest survival but a lower freedom from $\mathrm{CAV}$ in the re-transplanted heart. Early time-related risk of mortality after re-transplantation was similar to that after primary transplantation; however, late-phase time-related risk of mortality was higher. Long-term morbidities were significantly more common after re-transplantation than with primary transplantation including CAV $(p<0.001)$, late rejection $(p<0.001)$, and late renal dysfunction $(p<0.001)$. Malignancy after re-transplantation was, however, comparable to that for primary transplantation $(p=0.52)$.

\section{Special populations}

\section{Restrictive cardiomyopathy}

The clinical presentation of pediatric restrictive cardiomyopathy (RCM) varies, ranging from no symptoms to overt heart failure, syncope, or sudden death. Many of the clinical manifestations are the result of elevated filling pressures causing pulmonary edema, pulmonary hypertension, hepatomegaly, and peripheral edema. [33] Children with RCM have especially poor outcomes. In the largest cohort study to date, using data from the Pediatric Cardiomyopathy Registry, five-year survival from the diagnosis of RCM was $68 \%$. Transplantfree survival in children with pure RCM is worse than in children with a mixed RCM/HCM phenotype, with one- and
Fig. 8 Freedom from lymphoma by induction type in pediatric heart transplant recipients (used with permission from the Registry of the International Society of Heart and Lung Transplantation. J Heart Lung Transplant 2017;36:1037-79)

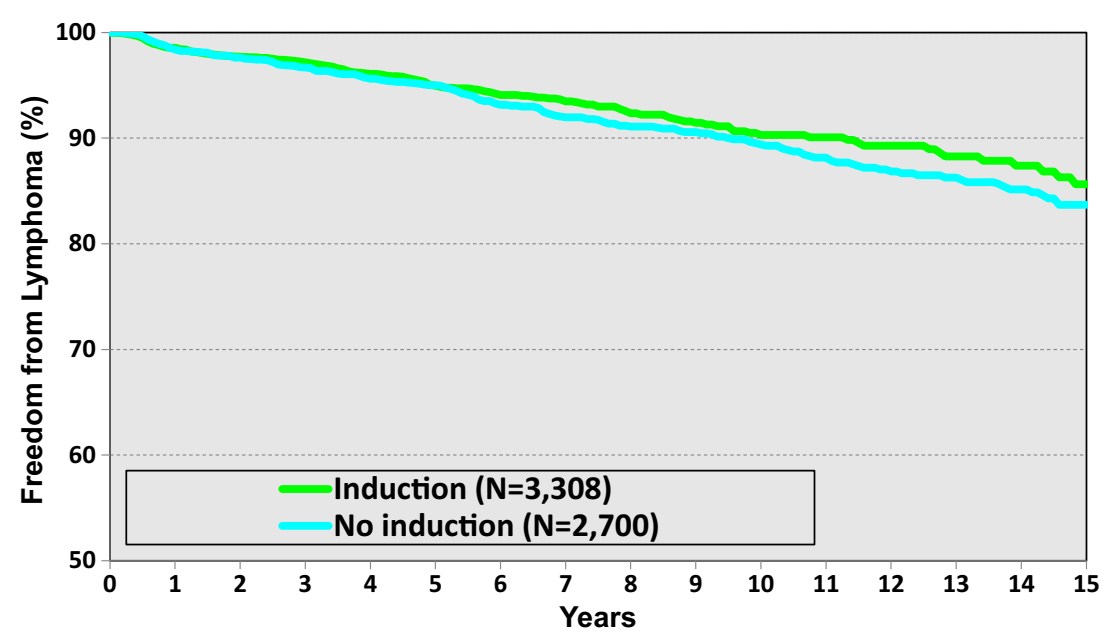


Fig. 9 Pediatric heart retransplants by year of transplantation (used with permission from the Registry of the International Society of Heart and Lung Transplantation. J Heart Lung Transplant 2017;36:1037-79)

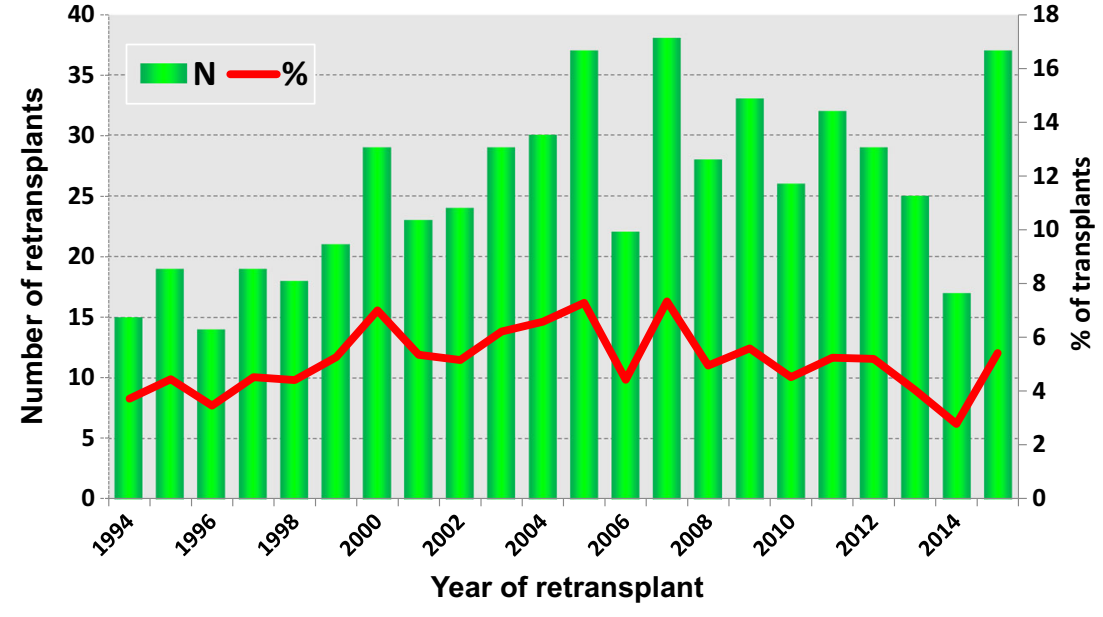

five-year survivals of $48 \%$ and $22 \%$ vs $65 \%$ and $43 \%$, respectively. Overall, outcomes were worse than for all other forms of cardiomyopathy. [34]

With limited medical therapies, poor mechanical support options, and the absence of risk factors that consistently predict disease progression or sudden death, early consideration for heart transplantation has been promoted for RCM patients. [33] A study using PHTS data showed that overall waitlist mortality for children with RCM was low at only 9\%. [35] Risk factors for death while waiting included younger age $(p<0.001)$, ventilator dependence $(p<0.001)$, higher status $(p<0.001)$, and inotrope usage $(p<0.001)$. Use of multiple support devices at listing (ventilator, extracorporeal membrane oxygenation, ventricular assist device, intraaortic balloon pump) was also an important risk factor for early death while waiting (relative risk; $9.01, p<0.0001)$. Survival after listing was $63 \%$ at 10 years, which was similar to survival for noncardiomyopathy patients $(p<0.01)$ and those with other forms of cardiomyopathy. [35]

\section{Fontan patients}

Staged palliation culminating in the Fontan procedure remains the standard of care of children with complex congenital heart disease and single ventricle physiology. Progressive heart failure, unfortunately, develops over time in a considerable portion of these patients and they will ultimately require heart transplantation. Historically, patients with prior Fontan procedure have been considered at higher risk with decreased postheart transplant survival compared with other forms of congenital heart disease or cardiomyopathies. [36]

More recent data out of PHTS compared Fontan patients who underwent heart transplantation between the early era (1993 to 2006, $n=150$ ) and the late era (2007 to 2014, $n=$ 252). [7] Post-heart transplantation survival and pre-heart transplantation characteristics were compared among eras and with non-Fontan congenital heart defect patients. Postheart transplantation Fontan patient survival significantly improved from the early to late era $(p=0.02)$, particularly in the early phase with 1 -year survival of $77 \%$ in the early era and
Fig. 10 Pediatric heart retransplants by inter-transplant interval and recipient age (used with permission from the Registry of the International Society of Heart and Lung Transplantation. J Heart Lung Transplant 2017;36:103779)

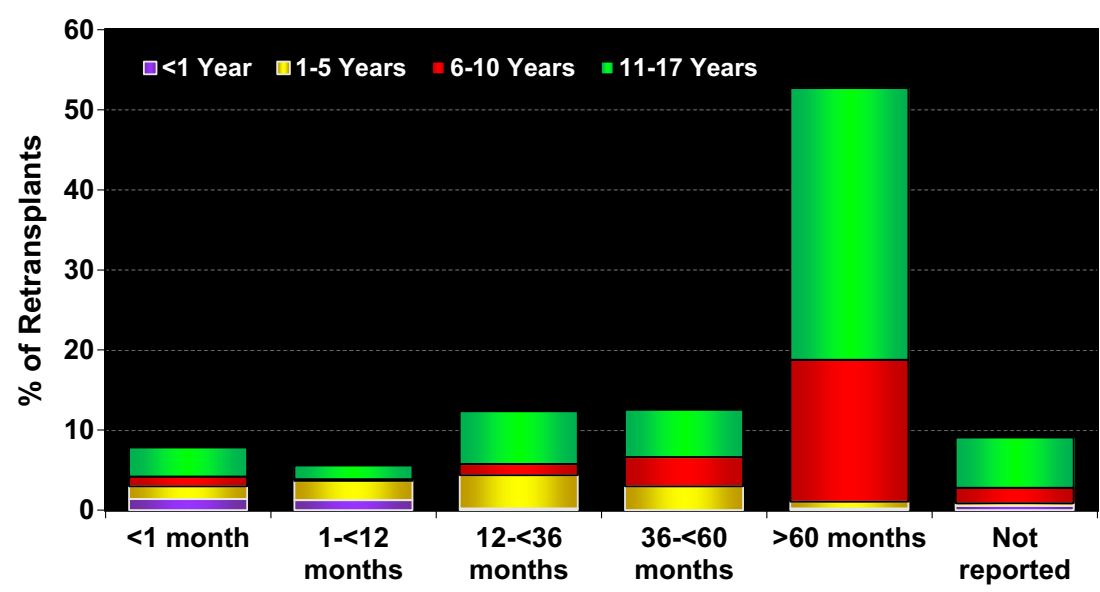

Time Between Previous and Current Transplant 
$89 \%$ in the late era. Late era non-Fontan CHD patients' 1-year post-heart transplantation survival was similar to Fontan patients at $92 \%$. Although, in the late era, Fontan patients were more likely to require inotropic support, have protein-losing enteropathy and failure to thrive at the time of transplant, they were less likely to require ventilator support, which, along with earlier year of heart transplantation, were the only risk factors for death in the multivariate analysis.

\section{ABO-incompatible heart transplantation}

The immaturity and malleability of the infant immune system has allowed for the evolution of ABO-incompatible heart transplantation. This is now accepted as the standard of care both from a waitlist mortality and a post-transplant survival and outcomes perspective. In fact, failure to list for an $\mathrm{ABO}$ incompatible transplant and high clinical status have emerged as the main factors associated with mortality. [2] Research continues to show equivalent outcomes for ABO-incompatible heart transplantation with respect to rejection, co-morbidities, $\mathrm{CAV}$, and graft survival. PHTS data showed equivalent 1-year survival and freedom from rejection in ABO-incompatible and compatible infant recipients in both center and risk-adjusted analysis. [5] Recent literature in both cardiac and renal transplantation has shown that successful ABO-incompatible transplants can be done in older patients, including adults even when significant isohemagglutinins are present. [2]

A single-center study reviewed 12 out of 30 patients who underwent $\mathrm{ABO}$-incompatible heart transplantation between 2000 and 2013 with pre-existing isohemagglutinin titers of $>$ 1:16. [37] The median age was 14.9 (range 9.8 to 107.3) months and median weight was 9.6 (range 7.6 to 25 ) $\mathrm{kg}$. Pre-transplant diagnosis was cardiomyopathy in $67 \%$ and congenital heart disease in $33 \%$. The highest pre-transplant isohemagglutinin titer was 1:256 in 2 patients. Four patients (33\%) had early antibody-mediated rejection (AMR) within 2 weeks of transplant. Successful management of these patients included the use of rituximab, bortezomib, immunoadsorption, and eculizumab. Three patients died but no deaths were associated with high isohemagglutinin titers. Although longer-term outcomes remain to be seen, this study demonstrated that cardiac transplantation was not only possible but has good outcomes in patients with higher isohemagglutinin titers when managed appropriately. [37]

\section{Sensitized patients}

The production of antibodies to human leukocyte antigen (HLA) prior to transplantation has increasingly been recognized as an important contributor to clinical outcomes in heart transplantation. [38] A number of risk factors for the development of anti-HLA antibodies prior to heart transplantation have been identified including transfusion of blood products, previous organ transplantation, history of pregnancy, previous cardiac surgery (especially surgery requiring exposure to homograft materials for surgical reconstruction), and the implantation of ventricular assist devices for mechanical support. [39]

Currently, anywhere from 15 to $30 \%$ of pediatric patients listed for heart transplantation are reported to be sensitized with a panel reactive antibody (PRA) $\geq 10 \%$, and this number has been increasing in recent years. [40] Data from the ISHLT registry revealed that patients with congenital heart disease were more likely to have some degree of sensitization compared with cardiomyopathy patients, approaching nearly $40 \%$ in adolescents. [1] Cardiomyopathy patients were not free, however, from the risk of developing anti-HLA antibodies. Pediatric data with respect to the role of elevated PRA on post-transplant outcomes is limited with conflicting results. Allosensitization, as measured by elevated PRA level, was not associated with long-term survival in the ISHLT registry data (Figs. 11 and 12). [1] Elevated PRA was not found to affect 30 -day survival $(25 \%$ vs. $7.9 \%, p=0.178)$ but was associated with higher overall mortality (50\% vs. $15.4 \%, p=0.043)$ in a small cohort of pediatric heart transplant recipients. [41] A larger, single-center study assessed outcomes of transplant recipients with either positive PRA before transplant or positive retrospective crossmatch. [22] There was no difference in graft survival between those patients with elevated PRA results and those who were negative; however, those patients with a positive retrospective crossmatch exhibited a worse overall graft survival $(p<0.015)$ despite no difference in time to cellular rejection, rejection grade, the number of rejection episodes, or infections. A PHTS study looking at the association between elevated PRA and outcomes between January 1993 and December 2008 showed similar findings. [40] Six-month survival post-transplant was lower $(77 \%$ vs. $93 \%, p<0.001)$ in patients who exhibited a PRA $\geq 50 \%$ compared with those with a PRA $<10 \%$. Those with elevated PRA who had a negative prospective crossmatch had no difference in survival compared with those without allosensitization, while those with elevated PRA and a positive prospective crossmatch had a survival disadvantage at 1 -year post-transplant $(p=0.0004)$. This study did not find any significant association between PRA levels and time to first rejection or development of CAV.

It is clear that sensitization is an issue that is increasing in frequency in the pediatric heart transplant population. The overall significant and clinical relevance of the antibodies detected remains uncertain. Listing these patients for transplant with the requirement for a negative crossmatch may decrease the risks post-transplant but at the expense of increased mortality while on the waitlist. $[22,38,40]$

\section{Extracorporeal membrane oxygenation}

Mechanical circulatory support for the failing heart has been used a bridge to heart transplantation in the pediatric 
Fig. 11 Kaplan-Meier survival by PRA in pediatric heart transplant patients with initial diagnosis of CHD (used with permission from the Registry of the International Society of Heart and Lung Transplantation. J Heart Lung Transplant 2017;36:1037-79)

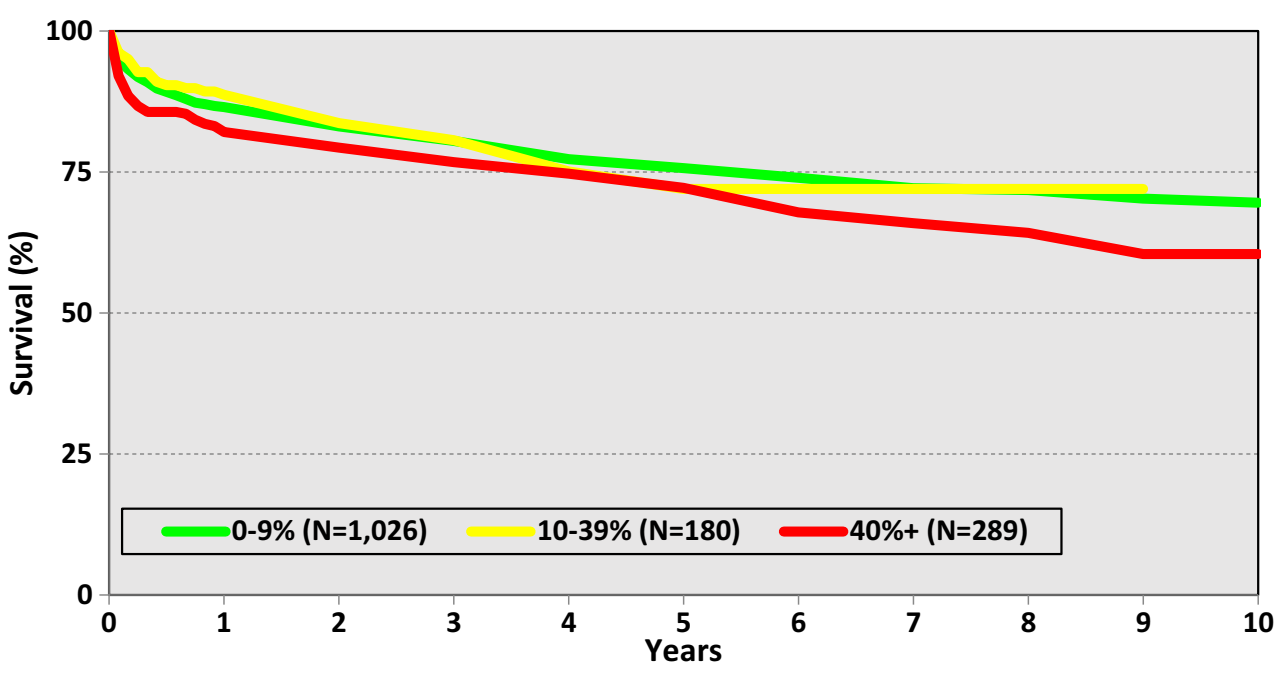

population with ECMO as the primary means of support. ECMO is associated with many complications, including thromboembolic events, bleeding, immobilization, infection, end-organ dysfunction, and risk of neurologic impairment. [42] As well, bridge to transplant on ECMO has been associated with high waitlist mortality and a poor survival to hospital discharge.

PHTS analyzed all patients listed for heart transplantation between 1993 and 2013 to determine the effect of ECMO support at the time of listing and the time of transplant on waitlist mortality and post-transplant outcomes. [43] Patients on ECMO support at the time of transplant had a significantly shorter waitlist duration ( 24 vs. 93 days, $p<0.0001$ ). Survival at 12 months after listing was worse in patients on ECMO at listing (50\%) compared with VAD at listing $(76 \%)$ or not on ECMO or VAD at listing $(76 \%, p<0.0001)$. Survival after heart transplantation was worse in patients who underwent transplantation from ECMO (3 years: 64\%) compared to VAD at transplantation (3 years: $84 \%$ ) or not on ECMO/ VAD at transplantation ( 3 years: $85 \%, p<0.001$ ). Patients transplanted from ECMO at age $<1$ year had the worst survival. More recent PHTS data showed a mean waitlist duration of 1 month for patients who underwent transplantation from ECMO support. [27]

Current organ allocation algorithms have been developed to direct hearts to the sickest recipients in order to mitigate death while waiting. The above results shed light that the prioritization of donor hearts to children waitlisted on ECMO warrants careful consideration.

\section{Ventricular assist devices}

Pediatric mechanical support has become an important therapy in the management of pediatric heart failure as a bridge to transplantation. This has led to improved waitlist survival at the risk of development of a number of complications, including bleeding, neurologic injury, infection, and device malfunction. In adults, these VAD- associated complications have been shown to decrease survival to transplant. [44] A pediatrics linkage analysis between PediMACS, the pediatric-specific subgroup of the
Fig. 12 Kaplan-Meier survival by PRA in pediatric heart transplant patients with initial diagnosis of DCM (used with permission from the Registry of the International Society of Heart and Lung Transplantation. J Heart Lung Transplant 2017;36:1037-79)

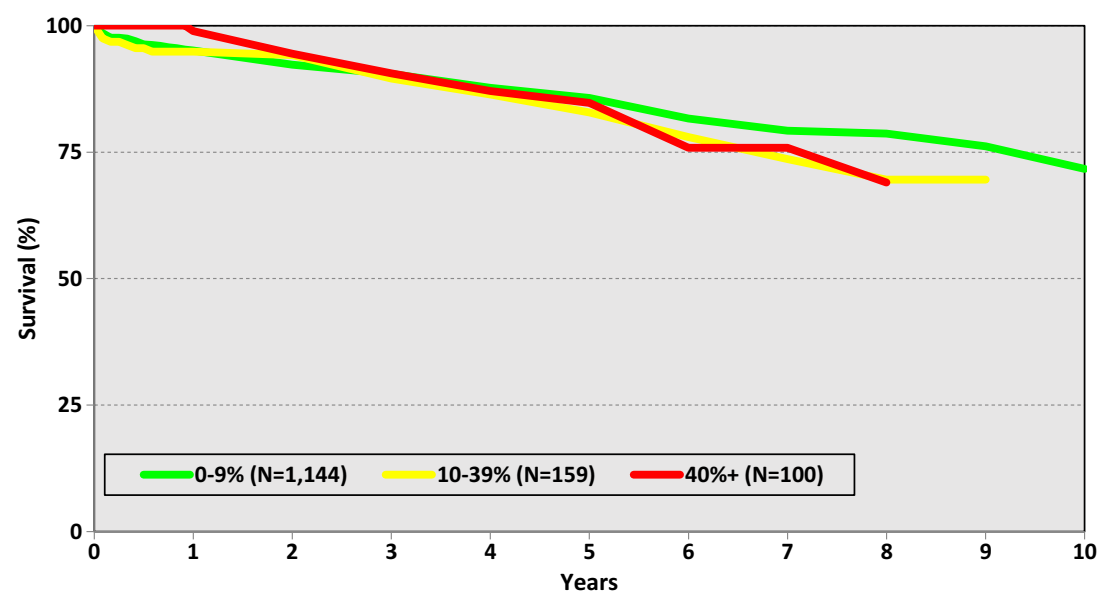


Interagency Registry for Mechanical Circulatory Support (INTERMACS), and PHTS was recently undertaken to determine the effects of VAD course on pediatric post-transplant outcomes. [45] Databases were linked between October 1, 2012 and December 31, 2015 identifying 147 transplanted VAD patients and comparing them to 630 patients without pre-transplant VAD support. At the time of VAD implant, $23 \%$ were INTERMACS Profile 1 (critical cardiogenic shock), 63\% were Profile 2 (progressive decline), and $14 \%$ were Profile 3 (stable but inotrope-dependent). The VAD cohort was older, larger, and less likely to have congenital heart disease $(p<0.0001)$. The VAD cohort had greater requirements for inotrope and ventilator support and increased liver and renal dysfunction $(p<0.0001)$, all of which normalized pre-transplant after device support. There were no differences in 1-year post-transplant survival ( $95 \%$ vs. $93 \%, p=0.3$ ), freedom from infection $(81 \%$ vs. $79 \%, p=0.9)$, or freedom from rejection ( $71 \%$ vs. $74 \%, p=0.87)$. Post-transplant length of stay was shorter by a median duration of 2 days in the VAD group (17 vs. $19, p=0.04$ ).

A more recent PHTS study looked at children supported to heart transplant between January 1, 1993 and December 31, 2015 with VAD or ECMO-to-VAD support. [27] Patients with a VAD as their first device were older, larger, more likely to have cardiomyopathy (80\%), and less likely to require inotropic support than both patients transitioned from ECMO-toVAD and patients not requiring mechanical support. In the VAD-only cohort, $79 \%$ underwent heart transplantation and $14 \%$ died, compared to $69 \%$ undergoing heart transplantation and $24 \%$ death in the ECMO-to-VAD cohort. Survival at 2 and 20 years showed no difference between VAD and no support ( 2 years: $75 \%$ vs. $80 \%$; 20 years: $55 \%$ vs. $54 \%$ ). Overall 2-year survival after listing was superior for patients with VAD support as their first device compared with patients who transitioned from ECMO to VAD $(p<0.01)$. Post-heart transplant outcomes were better for durable versus temporary VADs $(p<0.01)$ and for continuous versus pulsatile VADs $(p<0.01)$ from 2005 onward. Timing of VAD was not found to have an impact on post-heart transplant survival $(p=0.65)$.

It is evident that major advances have occurred in pediatric mechanical support, which have expanded the capability to bridge to heart transplant. Significant challenges remain and it appears that circumventing transplantation directly from ECMO via transition from ECMO-to-VAD or primary VAD insertion is associated with improved outcomes.

\section{Transition and adherence}

Transition of medical care is the process of transferring care from a pediatric practitioner to an adult practitioner. [46] The standard of transition was initially defined as "the purposeful, planned movement of adolescents and young adults with chronic physical and medical conditions from child-centered to adult-oriented health-care systems". [47] Over time, the definition has broadened to include more of the psychosocial aspect and has become "a purposeful, planned process that addresses the medical, psychological, and educational needs of adolescents and young adults with chronic physical and medical conditions as they move from child-centered to adult-oriented healthcare systems." [48] Heart transplant recipients need regular visits with a myriad of health care providers. [48] Graft survival has improved with more children surviving into adulthood. Despite this survival, medium-term graft losses for adolescent transplant recipients are worse than all other age groups, except for those more than 65 years of age, mostly as a result of poor adherence. [49] When transition is not done or not done effectively, a decline in attendance to medical follow-up to adult-centered clinics and accompanying increase in morbidity is commonly seen. [50,51]

Transition is not a single point in time, but rather, it is a process that involves many steps and resources. [48] Preparation involves health education, readiness assessment tools, creating a health passport, and promoting self-management. Administration resources include institutional support, a transition coordinator, and the development of an appropriate transition protocol. The actual transfer itself involves identifying an adult provider, meeting the adult practice and choosing a final specific transfer date. [48] There are a number of barriers to a successful transition including numerous team members, patient development and maturity, parents, pediatric and adult care providers, peers and high-risk behavior, patient and provider fears, and location. $[48,52]$ Despite these barriers, the evidence demonstrates that transition protocols and programs have measurable benefits for young patients and their parents with respect to improved follow-up, better disease control, and improved documentation. [48, 53]

Adherence in medicine most commonly refers to medication or drug compliance, but can also apply to attending appointments, completing required blood tests, self-care, selfdirected exercises, or therapy sessions. As mentioned above, medium-term graft losses for adolescent transplant recipients are worse than all other age groups, except for those more than 65 years of age, mostly as a result of poor adherence. [49] This finding has been confirmed in multiple studies including an assessment of a large national database recording all deceased solid-organ transplant recipients that found better graft survival for adolescents initially, followed by crossing of the lines, such that adolescents in all solid-organ transplant groups, including heart, had worse survival. [54] Non-adherence was found to be a significant risk factor for all cardiac causes of death, notable graft failure $(p=0.001)$ and rejection $(p<0.001)$ in a study using ISHLT registry data. [55] Given these findings, a number of interventions and trials have been piloted with a focus on improving adherence among adolescent transplant recipients. 
An adult-based randomized controlled trial tested the efficacy of a post-transplant medication adherence enhancing intervention on clinical outcomes. [56] Adult heart, liver, and lung transplant recipients who were $>1$ year post-transplant and on tacrolimus twice daily were randomly assigned to an intervention group or a control group. The intervention group received staged multicomponent tailored behavioral intervention, whereas the control group received usual medical care. Baseline demographics related to adherence were comparable. The intervention group had a $16 \%$ higher dosing adherence postintervention $(p<0.001)$, resulting in odds of adherence being five times higher in the intervention group. The effect was sustained at the end of follow-up. A similar pediatric-based randomized trial was done to assess the efficacy of a clinicbased adherence-promoting intervention in kidney transplant recipients. [57] Participants were assigned to either the 'TAKE-IT' intervention group or a control group and adherence was electronically monitored in all participants for 3 months to start. Participants in the intervention group could choose to receive text message, e-mail, and/or visual cue dose reminders and met with a coach at 3-month intervals where adherence data from the prior 3 months were reviewed. Action-focused problem solving was used to address adherence barriers selected as important by the participant. Participants assigned to the control group met with coaches at 3-month intervals but received no feedback about adherence data. Participants in the intervention group had significantly greater odds of taking prescribed medications (OR, 1.66; 95\% CI, 1.15-2.39) and taking medications at or near the prescribed time (OR, 1.74; 95\% CI, 1.21-2.50) than controls.

Many smaller pilot studies have been carried out around the implementation of various interventions including mentoring programs, group visits, technology-based approaches, text messages, and phone apps. These studies all aim to establish the feasibility and acceptability of various intervention approaches and whether adherence was increased and sustained over the study and follow-up period. It is undeniable that transition to adult care and medical adherence is a challenge that requires processes, interventions, and a large support network or team.

\section{Conclusions}

Pediatric heart transplantation is standard of care for children with end-stage heart failure. Outcomes in the most recent era are excellent resulting in a shift in focus towards medium-and long-term outcomes. Rejection, CAV, graft failure, and infection continue to be the major causes of death within the first 5 years post-transplant but have also shown vast improvements in survival and outcomes in the more recent eras. As well, renal dysfunction, malignancy, and the need for retransplantation remain as significant issues that will continue to require close follow-up and understanding of risk factors. Overall, pediatric heart transplantation continues to evolve and grow in order to bettercare for the diverse group of patients that reach end-stage heart failure during childhood.

\section{Compliance with ethical standards}

Not required being a review article.

Conflict of interest The authors declare that they have no conflict of interest.

\section{References}

1. Rossano JW, Cherikh WS, Chambers DC, et al. The Registry of the International Society for Heart and Lung Transplantation : Twentieth Pediatric Heart Transplantation Report - 2017 ; Focus Theme : Allograft ischemic time. J Heart Lung Transplant. 2017;36:1060-9.

2. Dipchand AI. Current state of pediatric cardiac transplantation. Ann Cardiothorac Surg. 2018;7:31-5.

3. Mehra M, Kobashigawa J, Starling R, et al. Listing criteria for heart transplantation: International Society for Heart and Lung Transplantation guidelines for the care of cardiac transplant candidates - 2006. J Heart Lung Transplant. 2006;25:1024- 42.

4. Ryan TD, Chin C. Pediatric cardiac transplantation. Semin Pediatr Surg. 2017;26:206-12.

5. Aziz TM, Burgess MI, El-Gamel A, et al. Orthotopic cardiac transplantation technique: a survey of current practice. Ann Thorac Surg. 1999;68:1242-6.

6. Kanani M, Hoskote A, Carter C, Burch M, Tsang V, Kostolny M. Increasing donor-recipient weight mismatch in pediatric orthotopic heart transplantation does not adversely affect outcome. Eur J Cardiothorac Surg. 2012;41:427-34.

7. Simpson KE, Pruitt E, Kirklin JK, et al. Fontan Patient Survival After Pediatric Heart Transplantation Has Improved in the Current Era. Ann Thorac Surg. 2017;103:1315-20.

8. Pietra BA, Kantor PF, Bartlett HL, et al. Early predictors of survival to and after heart transplantation in children with dilated cardiomyopathy. Circulation. 2012;126:1079-86.

9. Dipchand AI, Kirk R, Mahle WT, et al. Ten yr of pediatric heart transplantation: a report from the Pediatric Heart Transplant Study. Pediatr Transplant. 2013;17:99-111.

10. Ameduri RK, Zheng J, Schechtman KB, et al. Has late rejection decreased in pediatric heart transplantation in the current era? A multi-institutional study. J Heart Lung Transplant. 2012;31:980-6.

11. Gossett JG, Canter CE, Zheng J, et al. Decline in rejection in the first year after pediatric cardiac transplantation: a multi-institutional study. J Heart Lung Transplant. 2010;29:625-32.

12. Godown J, Harris MT, Burger J, Dodd DA. Variation in the use of surveillance endomyocardial biopsy among pediatric heart transplant centers over time. Pediatr Transplant. 2015;19:612-7.

13. Kobayashi D, Du W, L'Ecuyer TJ. Predictors of cardiac allograft vasculopathy in pediatric heart transplant recipients. Pediatr Transplant. 2013;17:436-40.

14. Pahl E, Naftel DC, Kuhn MA, et al. The impact and outcome of transplant coronary artery disease in a pediatric population: a 9-year multi-institutional study. J Heart Lung Transplant. 2005;24:645-51.

15. Chin C, Gamberg P, Miller J, Luikart H, Bernstein D. Efficacy and safety of atorvastatin after pediatric heart transplantation. J Heart Lung Transplant. 2002;21:1213-7. 
16. Mahle WT, Vincent RN, Berg AM, Kanter KR. Pravastatin therapy is associated with reduction in coronary allograft vasculopathy in pediatric heart transplantation. J Heart Lung Transplant. 2005;2:636.

17. Greenway SC, Butts R, Naftel DC, et al. Statin therapy is not associated with improved outcomes after heart transplantation in children and adolescents. J Heart Lung Transplant. 2016;35:457-65.

18. Eisen HJ, Tuzcu EM, Dorent R, et al. Everolimus for the prevention of allograft rejection and vasculopathy in cardiac-transplant recipients. N Engl J Med. 2003;349:847-58.

19. Keogh A, Richardson M, Ruygrok P, et al. Sirolimus in de novo heart transplant recipients reduces acute rejection and prevents coronary artery disease at 2 years: a randomized clinical trial. Circulation. 2004;110:2694-700.

20. Arora S, Ueland T, Wennerblom B, et al. Effect of everolimus introduction on cardiac allograft vasculopathy-results of a randomized, multicenter trial. Transplantation. 2011;92:235-43.

21. Kindel SJ, Pahl E. Current therapies for cardiac allograft vasculopathy in children. Congenit Heart Dis. 2012;7:324-35.

22. Wright EJ, Fiser WP, Edens RE, et al. Cardiac transplant outcomes in pediatric patients with pre-formed anti-human leukocyte antigen antibodies and/or positive retrospective crossmatch. J Heart Lung Transplant. 2007;26:1163-9.

23. Feingold B, Zheng J, Law YM, et al. Risk factors for late renal dysfunction after pediatric heart transplantation: a multi-institutional study. Pediatr Transplant. 2011;15:699-705.

24. Dipchand AI, Rossano JW, Edwards LB, et al. The Registry of the International Society for Heart and Lung Transplantation: Eighteenth Official Pediatric Heart Transplantation Report - 2015; Focus Theme: Early Graft Failure. J Heart Lung Transplant. 2015;34:1233-43.

25. Rostad CA, Wehrheim K, Kirklin JK, et al. Bacterial infections after pediatric heart transplantation: epidemiology, risk factors and outcomes. J Heart Lung Transplant. 2017;36:996-1003.

26. Mahle WT, Fourshee MT, Naftel DM, et al. Does cytomegalovirus serology impact outcome after pediatric heart transplantation? J Heart Lung Transplant. 2009;28:1299-305.

27. Dipchand AI, Kirk R, Naftel DC, et al. Ventricular assist device support as a bridge to transplantation in pediatric patients. J Am Coll Cardiol. 2018;72:402-15.

28. Ng B, Dipchand A, Naftel D, et al. Outcomes of Pneumocystis jiroveci pneumonia infections in pediatric heart transplant recipients. Pediatr Transplant. 2011;15:844-8.

29. Webber SA, Naftel DC, Fricker FJ, et al. Lymphoproliferative disorders after paediatric heart transplantation: a multi-institutional study. Lancet. 2006;367:233-9.

30. Chinnock R, Webber SA, Dipchand AI, Brown RN, George JF. A 16-year multi-institutional study of the role of age and EBV status on PTLD incidence among pediatric heart transplant recipients. Am J Transplant. 2012;12:3061-8.

31. Chin C, Naftel D, Pahl E, et al. Cardiac re-transplantation in pediatrics: a multi-institutional study. J Heart Lung Transplant. 2006;25: $1420-4$.

32. Conway J, Manlhiot C, Kirk R, Edwards LB, McCrindle BW, Dipchand A. Mortality and morbidity after retransplantation after primary heart transplant in childhood: an analysis from the registry of the International Society for Heart and Lung Transplantation. J Heart Lung Transplant. 2014;33:241-51.

33. Lee TM, Hsu DT, Kantor P, et al. Pediatric cardiomyopathies. Circ Res. 2017;121:855-73.

34. Webber SA, Lipshultz SE, Sleeper LA, et al. Outcomes of restrictive cardiomyopathy in childhood and the influence of phenotype: a report from the pediatric cardiomyopathy registry. Circulation. 2012;126:1237-44.

35. Zangwill SD, Naftel D, L'Ecuyer T, et al. Outcomes of children with restrictive cardiomyopathy listed for heart transplant: a multiinstitutional study. J Heart Lung Transplant. 2009;28:1335-40.

36. Voeller RK, Epstein DJ, Guthrie TJ, Gandhi SK, Canter CE, Huddleston CB. Trends in the indications and survival in pediatric heart transplants: a 24-year single-center experience in 307 patients. Ann Thorac Surg. 2012;94:807-16.

37. Irving CA, Gennery AR, Carter V, et al. ABO-incompatible cardiac transplantation in pediatric patients with high isohemagglutinin titers. J Heart Lung Transplant. 2015;34:1095-102.

38. Conway J, Dipchand AI. Challenges with sensitized recipients in pediatric heart transplantation. Clinics. 2014;69:17-21.

39. Shaddy RE, Fuller TC. The sensitized pediatric heart transplant candidate: causes, consequences, and treatment options. Pediatr Transplant. 2005;9:208-14.

40. Mahle WT, Tresler MA, Edens RE, et al. Allosensitization and outcomes in pediatric heart transplantation. J Heart Lung Transplant. 2011;30:1221-7.

41. Jacobs JP, Quintessenza JA, Boucek RJ, et al. Pediatric cardiac transplantation in children with high panel reactive antibody. Ann Thorac Surg. 2004;78:1703-9.

42. Ibrahim AE, Duncan BW, Blume ED, Jonas RA. Long-term follow-up of pediatric cardiac patients requiring mechanical circulatory support. Ann Thorac Surg. 2000;69:186-92.

43. Dipchand AI, Mahle WT, Tresler M, et al. Extracorporeal Membrane Oxygenation as a Bridge to Pediatric Heart Transplantation. Effect on post-listing and post- transplanatation outcomes. Circ Hear Fail. 2015;8:960-9.

44. Kirklin JK, Naftel DC, Pagani FD, et al. Seventh INTERMACS annual report: 15,000 patients and counting. J Heart Lung Transplant. 2015;34:1495-504.

45. Sutcliffe DL, Pruitt E, Cantor RS, et al. Post-transplant outcomes in pediatric ventricular assist device patients: A PediMACS-Pediatric Heart Transplant Study linkage analysis. J Heart Lung Transplant. 2018:37:715-22.

46. Niwa K. Adults with congenital heart disease transition. Curr Opin Pediatr. 2015;27:576-80.

47. Blum RW, Garell D, Hodgman CH, et al. Transition from childcentered to adult health-care systems for adolescents with chronic conditions. A position paper of the society for adolescents medicine. J Adolesc Health. 1993;14:570-6.

48. Putschoegl A, Dipchand AI, Ross H, Chaparro C, Johnson JN. Transitioning from pediatric to adult care after thoracic transplantation. J Heart Lung Transplant. 2017;36:823-9.

49. McDonagh JE, Kaufman M. Transition from pediatric to adult care after solid organ transplantation. Curr Opin Organ Transplant. 2009; 14:526-32.

50. Saidi A, Kovacs AH. Developing a transition program from pediatric- to adult-focused cardiology care: practical considerations. Congenit Heart Dis. 2009;4:204-15.

51. Lugasi T, Achille M, Stevenson M. Patients' perspective on factors that facilitate transition from child-centered to adult-centered health care: a theory integrated metasummary of quantitative and qualitative studies. J Adolesc Heal. 2011;48:429-40.

52. Annunziato RA, Freiberger D, Martin K, Helcer J, Fitzgerald C, Lefkowitz DS. An empirically based practice perspective on the transition to adulthood for solid organ transplant recipients. Pediatr Transplant. 2014;18:794-802.

53. Kerkar N, Annunziato R. Transitional care in solid organ transplantation. Semin Pediatr Surg. 2015;24:83-7. 
54. Dharnidharka VR, Lamb KE, Zheng J, Schechtman KB, MeierKriesche HU. Across all solid organs, adolescent age recipients have worse transplant organ survival than younger age children: A US national registry analysis. Pediatr Transplant. 2015;19:471-6.

55. Vanderlaan RD, Manlhiot C, Edwards LB, Conway J, McCrindle BW, Dipchand AI. Risk factors for specific causes of death following pediatric heart transplant: an analysis of the registry of the International Society of Heart and Lung Transplantation. Pediatr Transplant. 2015; 19:896-905.

56. Dobbels F, De Bleser L, Berben L, et al. Efficacy of a medication adherence enhancing intervention in transplantation: The MAESTRO-Tx trial. J Heart Lung Transplant. 2017;36:499-508.
57. Foster BJ, Pai ALH, Zelikovsky N, et al. A randomized trial of a multicomponent intervention to promote medication adherence: The Teen Adherence in Kidney Transplant Effectiveness of Intervention Trial (TAKE-IT). Am J Kidney Dis. 2018;72:30-41.

Publisher's note Springer Nature remains neutral with regard to jurisdictional claims in published maps and institutional affiliations. 